\title{
Actitud hacia el aprendizaje de la Física de los estudiantes del tercer año de Educación Media General
}

\author{
Attitude towards the learning of Physics of the students of the third year \\ of General Middle Education \\ María Sabrina López Moreno sabrilop.21.12@gmail.com \\ Código ORCID: 0000-0003-3086-5438 \\ Unidad Educativa Creación San Pablo, Venezuela \\ Recibido septiembre 2019 | Arbitrado octubre 2019 | Publicado enero 2020
}

\begin{abstract}
Resumen
Palabras clave:

Actitud; aprendizaje; física

El presente estudio tuvo como objetivo fundamental distinguir la actitud hacia la Física de los alumnos de 3er año del Ciclo Básico San Pablo, estado Yaracuy- Venezuela. Esta investigación fue de tipo descriptivo con diseño de campo, no experimental y transeccional. La población objeto de estudio estuvo conformada por 128 estudiantes de tercer año de educación media general del Ciclo Básico San Pablo, y la muestra por 08 estudiantes, que se obtuvo por un muestreo aleatorio simple. En la recolección de la información se utilizó la encuesta, conformada por 12 ítems arrojando una confiabilidad a través del Alfa de Cronbach de 0,879, considerado en su escala de estimación una magnitud muy alta, que permitió realizar el diagnóstico de la actitud que poseen los estudiantes hacia la Física. Se concluyó que existe una tendencia a una actitud positiva hacia la Física por parte de los estudiantes del tercer año.
\end{abstract}
Abstract
Keywords:
Attitude;
earning;
physical
The main objective of the present study was to distinguish the attitude towards Physics of the 3rd year students of the Basic Cycle San Pablo, Yaracuy state- Venezuela. This research was descriptive in field design, non-experimental and transectional. The study population consisted of 128 third-year general education students from the Ciclo Básico San Pablo, and the sample of 08 students, which was obtained by simple random sampling. In the collection of information, the survey was used, consisting of 12 items, yielding reliability through the cronbach's alpha of 0.879 , considered a very high magnitude on its estimation scale, which made it possible to diagnose the attitude that students possess towards Physics. It was concluded, there is a tendency towards a positive attitude towards Physics by third year students. 


\section{INTRODUCCIÓN}

La importancia social de la ciencia aunada a la generalización de la educación trae consigo una necesidad de formar científicamente al hombre como una forma de transformarlos en garantes del desarrollo de un país. De allí que la generalización de la educación y el auge de las investigaciones sobre la optimización del proceso enseñanza aprendizaje de las ciencias deberían de ir acompañadas de una mejor alfabetización científica y tecnológica de la sociedad.

Por ello, Solbes, Monserrat y Furió (2007) consideran que "la crisis de la enseñanza en la educación secundaria alcanza en este momento a la mayoría de los países, especialmente en las áreas de ciencias" (p.92); razón por la cual se deben analizar los factores causantes de dicha crisis; para de esta forma lograr mejorar el aprendizaje de las ciencias, especialmente la física en los estudiantes de educación básica.

Por su parte, en Venezuela, los cursos de ciencias naturales y física en educación media son obligatorios. Además, la mayoría de los jóvenes, al pasar a este nivel educativo, estudian la mención ciencias debido a la reducida oferta educacional en otras áreas, por lo que ésta se convierte casi en obligatoria. Así mismo, a nivel universitario, en la carrera de física y en la docencia en física cuentan con una matrícula considerablemente baja. Todo esto producto de la apatía que existe por parte de los estudiantes hacia la física bien sea por factores psicológicos productos de los tabúes creados alrededor de dicha asignatura cuando desde el pasado era incluida como una de las materias consideradas popularmente con las tres María.

Es así como, Fensham en Vázquez y Manassero (2007) señalan que la enseñanza de las ciencias y la educación científica debe afrontar las inapropiadas y negativas actitudes que tiene el estudiantado hacia la ciencia, y más específicamente, la falta de interés hacia la ciencia en la escuela; no obstante, esta situación debe ser abordada de forma tal que se logre la debida motivación de la comunidad estudiantil, a través de la aplicación de diversas estrategias que propicien el interés o actitudes positivas hacia la física.

En este sentido, el estudio de las actitudes puede suscitar instancias que permitan hacer los aprendizajes significativos y por tanto, mejorar su calidad, relacionándolos eventualmente con los contenidos en Física, pues para Fensham (2004), el principal problema de la enseñanza son las actitudes desfavorables de las y los estudiantes hacia la ciencia y más específicamente, la falta de interés que existe hacia la misma en la instituciones educativas; producto de las debilidades que puedan presentar los docentes responsables de impartir las asignaturas correspondientes a las ciencias, tales como física y química; aunado a la carencia de laboratorios dotados y adecuados para llevar a cabo actividades prácticas que propicien una mejor comprensión de los contenidos de dichas asignaturas.

Por su parte González, Morales y Pino (2012) señala que:

En general las actitudes,
motivaciones e intereses de los
alumnos hacia la ciencia, han sido
objeto de numerosos estudios a
nivel internacional. Sí bien, existe
una gran trayectoria al respecto,
es en los últimos años que ha
captado la atención de los
investigadores como un factor
determinante del rendimiento
escolar. (p.6)

De acuerdo a lo expuesto por los autores, las actitudes adoptadas por estudiantes ante el aprendizaje de cualquier asignatura, especialmente las relacionadas con las ciencias, repercutirá directamente su rendimiento académico, así como en la consolidación de los conocimientos requeridos para su formación integral.

En otro orden de ideas, en lo que respecta a la estructura del interés hacia la física, se piensa 
que el estudiante, incorpora y excluye unidades de interés a dicha estructura, de acuerdo a sus experiencias dentro y fuera de la institución educativa hasta alcanzar una estructura compleja conformada por múltiples relaciones que estipulan su interés hacia la disciplina.

Para Nachtigall (1985), una forma de lograr que los alumnos se motiven verdaderamente y puedan trabajar en la construcción de un aprendizaje significativo es planificando clases que no resulten tediosas, y evitando caer en una especie de activismo, donde pareciera que los estudiantes estuviesen haciendo muchas cosas, pero que significan poco para ellos, ya que a veces se dedican a seguir indicaciones, y ya que no se ha creado en ellos ningún conflicto, no hay hipótesis que probar.

Por su parte, Castellano (2013) considera que los profesores que enseñan física afrontan la dificultad que presentan los estudiantes en el proceso de aprendizaje de esta asignatura, con una complicación intrínseca debida al nivel de abstracción, el grado de sistematización y el lenguaje altamente formalizado en que se expresa la misma.

En este orden de ideas, Nachtigall (1985) señala algunos aspectos que intervienen en la situación actual de la enseñanza de la física: (a) La física, como asignatura que se enseña en las escuelas, resulta antipática, (b) Los planes de estudio no consiguen una aplicación más amplia, (c) El material de enseñanza no se ajusta a las demandas de la investigación en el campo de la psicología cognitiva, (d) En la enseñanza oral, la física se presenta como un conjunto de procedimientos formales, que la mayor parte de los estudiantes son incapaces de aprender porque aún no han alcanzado la capacidad de pensamiento apropiada, (e) Un aspecto particular del material demasiado extenso y demasiado abstracto, que no es de importancia evidente, es la rapidez con que se presenta en clase, (f) En la formación de profesores, existe una gran distancia entre las lecciones teóricas y abstractas que los aspirantes a profesores reciben en las universidades y la enseñanza práctica real que ellos tienen que realizar.

En concordancia con lo antes planteado, en el Ciclo Básico San Pablo, ubicado en el municipio Arístides Bastidas, estado Yaracuy, se ha podido constatar, a través de entrevistas informales con el personal docente, que debido a los cambios curriculares que se han suscitado en el año escolar 2016-2017; los profesores que imparten la asignatura física manifestaron que requieren de capacitación para llevar a cabo en proceso de enseñanza; lo cual conlleva a la incomprensión por parte de los estudiantes, quienes se muestran apáticos y desmotivados a hacerse partícipe de su aprendizaje.

Todo esto repercute en el bajo rendimiento de los alumnos de 3er año, quienes rechazan asistir a las clases de física; manifestando no entender las fórmulas y los despejes, por lo que se les dificulta la resolución de los problemas. En este orden de ideas, se podría considerar como un factor que interviene en dicha problemática, el hecho de que los docentes que imparten la asignatura en el 3er año de educación básica no son especialistas en física, lo que limitaría la aplicación de estrategias y recursos que faciliten el aprendizaje de los escolares en esta área del desarrollo del conocimiento físicos.

Por todo lo antes expuesto se hace necesario conocer la actitud de los estudiantes ante el aprendizaje de la física; en virtud de ello surge como objetivo general: Distinguir la actitud hacia la física de los alumnos de 3er año del Ciclo Básico San Pablo estado Yaracuy.

Para lograr este objetivo se realizaron cada uno de los siguientes objetivos específicos, los cuales fueron: 1. Establecer los componentes cognoscitivos de los estudiantes de 3er año hacia la física. 2. Identificar los componentes afectivos de los estudiantes de 3er año hacia la física.3. Precisar los componentes conductuales de los estudiantes de 3er año hacia la física.

En el marco de reconocer la importancia que tuvieron los estudios previos a la investigación se acota que, en primera instancia, 
se tiene el estudio realizado en Argentina para el Instituto de Investigaciones en Educación en las Ciencias Experimentales, FFHA-UNSJ por Morales; Mazzitelli y Olivera (2015) titulado La enseñanza y el aprendizaje de la Física y de la Química en el nivel secundario desde la opinión de estudiantes; en el cual presentaron algunos de los resultados alcanzados en un estudio realizado con docentes y alumnos de Física y de Química, desde el enfoque de las representaciones sociales (RS).

En este sentido, partieron de las dificultades que se presentan en el aula de Ciencias Naturales, que se evidencian, entre otros indicadores, a través del desinterés por aprender ciencias, el bajo rendimiento de los alumnos, la falta de participación y la disminución del número de estudiantes que siguen carreras afines a la Física y a la Química. Teniendo en cuenta lo expuesto generamos un espacio de reflexión sobre esta problemática, para docentes de Física y de Química y llevamos adelante una serie de actividades, entre ellas la indagación de las RS de sus alumnos.

La Actitud es considerada una forma de motivación social que predispone la acción de un individuo hacia determinados objetivos o metas. La actitud designa la orientación de las disposiciones más profundas del ser humano ante un objeto determinado. Existen actitudes personales relacionadas únicamente con el individuo y actitudes sociales que inciden sobre un grupo de personas.

Por su parte, Álvarez; Cuéllar; López; Adrada; Anguiano; Bueno; Comas y Gómez (2014) consideran que las actitudes, conjuntamente con la personalidad, la motivación, las expectativas de cada persona, la experiencia sociocultural o la ansiedad, se engloban dentro de las denominadas variables afectivas de aprendizaje.

Por ello, a lo largo de la vida, las personas adquieren experiencia y forman una red $u$ organización de creencias características, entendiendo por creencia la predisposición a la acción. La actitud engloba un conjunto de creencias, todas ellas relacionadas entre sí y organizadas en torno a un objeto o situación. Las formas que cada persona tiene de reaccionar ante cualquier situación son muy numerosas, pero son las formas comunes y uniformes las que revelan una actitud determinada.

En este sentido, el concepto de actitud es básico en dos campos: en psicología social y en la teoría de la personalidad. La actitud social es compartida y favorece los intereses sociales por encima de los individuales. En concordancia con lo expuesto, en cuanto a la temática que se aborda en el presente estudio, la actitud corresponde a la predisposición a la implementación del Proyecto Canaima para optimizar el proceso de enseñanza aprendizaje.

De allí, que se considera que la actitud está conformada por los siguientes componentes:

a. Componente Cognoscitivo: para que exista una actitud, es necesario que se dé también una representación cognoscitiva del objeto. Está formado por las percepciones y creencias hacia un objeto, así como por la información que se tiene sobre un objeto. Los objetos no conocidos o sobre los que no se posee información no pueden generar actitudes. La representación cognoscitiva puede ser vaga o errónea, en el primer caso el afecto relacionado con el objeto tenderá a ser poco intenso; cuando sea errónea no afectará para nada a la intensidad del afecto.

b. Componente Afectivo: es el sentimiento en favor o en contra de un objeto social. Es el componente más característico de las actitudes. Aquí radica la diferencia principal con las creencias y las opiniones que se caracterizan por su componente cognoscitivo.

En este sentido, la emoción es un término empleado frecuentemente como sinónimo de sentimientos y que en psicología se emplea para denominar una reacción que implica determinados cambios fisiológicos, tales como la aceleración o la disminución del ritmo del pulso, la disminución o el incremento de la actividad de ciertas glándulas, o un cambio de la 
temperatura corporal. Todo ello estimula al individuo, o alguna parte de su organismo, para aumentar su actividad. Las tres reacciones primarias de este tipo son la ira, el amor, y el miedo, que brotan como respuesta inmediata a un estímulo externo, o son el resultado de un proceso subjetivo, como la memoria, la asociación o la introspección.

Donde la ira constituye la pasión del alma, que causa indignación y enojo; mientras que el miedo es una perturbación angustiosa del ánimo por un riesgo o daño real o imaginario, siendo en el caso en estudio la actitud ante la implementación de nuevas tecnologías.

c. Componente Conductual: es la tendencia a reaccionar hacia los objetos de una determinada manera. Es el componente activo de la actitud.

En concordancia con lo expuesto, se tiene que la conducta es un modo de ser del individuo y conjunto de acciones que lleva a cabo para adaptarse a su entorno. La conducta es la respuesta a una motivación en la que están involucrados componentes psicológicos, fisiológicos y de motricidad. La conducta de un individuo, considerada en un espacio y tiempo determinados, se denomina "comportamiento".

Dentro de las bases filosóficas de la investigación se consideró la Teoría del Aprendizaje Social, donde uno de los teóricos que sustenta el presente trabajo es Bandura (1963), quien utiliza la Teoría del aprendizaje social también denominada Teoría Cognitiva, la cual señala, que la conducta humana es aprendida, según las oportunidades $\mathrm{y}$ experiencias proporcionadas por su ambiente. Así, la conducta social y las reglas sociales son aprendidas, a través, de la observación al atender lo que otros dicen y observan las consecuencias de sus acciones.

De esta manera, a través del uso del juego como herramienta didáctica, de acuerdo con el contexto de la presente investigación, el niño y niña van a consolidar el desarrollo psicomotor. Finalmente, como resumen del análisis de las teorías que con referencia al juego sustentan la investigación, se citan las de Vigotsky (1981), quien plantea, como parte del Desarrollo Próximo, que para conocer el desarrollo del niño y la niña, es necesario comprobar primero el nivel efectivo y real, que consiste en el "nivel de desarrollo de las funciones psicointelectivas que se ha conseguido como resultado de un específico proceso de desarrollo, ya realizado" (p. 33).

También analiza el nivel de la zona de desarrollo próximo o potencial, que genera un nuevo desarrollo que cambia los procesos intelectuales del individuo y que permite estimar la diferencia entre el nivel real de desarrollo, entendido como la capacidad de resolver independientemente un problema, y el nivel de desarrollo potencial, determinado mediante la resolución de problemas bajo la guía de un adulto o de otro actor mediador, así la mediación está vinculada con el concepto de "zona de desarrollo próximo".

En ese sentido, y de acuerdo con el análisis realizado por Martínez (2004), para este autor “...el juego es una actividad social, en la cual gracias a la cooperación con otros niños, se logran adquirir papeles o roles que son complementarios al propio." (p. 9). Es por ello, que las teorías antes expuestas, constituyen básicamente el sustento, no solamente de la presente investigación, sino también de las Bases Curriculares en cuanto al desarrollo de los aspectos lúdicos en el mismo, para lo cual se deberán diseñar las estrategias más adecuadas que permitan al docente cumplir los propósitos planteados.

También se fundamentó la investigación en la Teoría Ecológica donde la calidad de un conocimiento está en relación directa con lo que se elabora o estructura con el conocimiento previo del sujeto. El conocimiento, aquello que permite una adaptación al sujeto con su medio, o con los problemas que plantea la vida puede ser social, físico y cognitivo. 
Saber que se debe saludar a las personas mayores, es un conocimiento social; saber que el exponerse mucho tiempo al sol es dañino, es un conocimiento físico; saber determinar la diferencia en cantidad de un grupo de objetos con otro, estableciendo una relación mental o racional, es un conocimiento cognitivo.

Las operaciones que realiza el niño con los objetos, a su alrededor, va a determinar concepciones en términos mentales las que se logran no a partir de los objetos, sino a partir del conocimiento de las acciones que realiza.

A partir de lo dicho, surge una interrogante: ¿Cómo se construyen las estructuras operatorias mentales? Las estructuras intelectuales se desarrollan por la actividad, comparación, ordenación y clasificación con los objetos físicos y las acciones corporales.

Por ejemplo, un niño que está ensartando cuentas está desarrollando estructuras abstractas usando esquemas de seriación y clasificación. Esta construcción de las estructuras se lleva a cabo de una manera compleja e imprevista. Es sabido, que se pueden establecer tres tipos de conocimientos elementales en el niño: el social, el físico y el cognitivo; éstos últimos podríamos incluirlos dentro de lo que llamamos conocimiento sensorial y conocimiento racional. Estos conocimientos a su vez se construyen con el aporte y combinación de cuatro factores: maduración, transmisión sexual, experiencia con objetos y equilibración.

La maduración produce el crecimiento y desarrollo orgánico, lo que permite que el sujeto, bajo condiciones de aprendizaje alcance mayores logros; sentarse, gatear, ponerse de pie y caminar, son conductas que exigen maduración biológica. La experiencia física, igualmente, se refiere a la relación con los objetos del entorno (juguetes, sonajas, biberón, entre otros).

La equilibración se refiere al paso de un estadio inferior a otro superior como consecuencia de los estados anteriores. A medida que el niño se desarrolla va logrando un nivel de equilibrio superior, advirtiéndose que la equilibración necesita de la abstracción, sea ésta simple o reflexiva. Al respecto, la Teoría Ecológica de Bronfenbrenner (1987), defiende la tesis de la "imposibilidad de comprender el desarrollo de las personas al margen de los contextos en que éste tiene lugar." (p. 113).

De esta manera, la perspectiva ecológica adoptada por Bronfenbrenner, permite considerar en un enfoque amplio el impacto que pueden tener los entornos en que el niño y niña desarrolla su actividad, como elemento de participación directa de influencia mediada en el aprendizaje y el desarrollo humano, al igual que la relación que se establece entre ambos, como plantea Solé (2002): “...hace una aproximación respetuosa y rigurosa al estudio de los contextos de desarrollo rompiendo estereotipos formalmente arraigados." (p.4).

La Ecología del Desarrollo Humano de Bronfenbrenner, explica la influencia directa que tiene el entorno social sobre el individuo en desarrollo, destacándose allí la interacción entre el individuo y el entorno social, como proceso generador de cambios, con relación al currículo como tal, quien señala desde la perspectiva de construcción cultural, la experiencia humana como punto de partida para organizar la práctica educativa, dándole así concreción al hecho pedagógico como praxis social.

La presente investigación se sustentó en un cuerpo de normativas legales, entre las que se encuentran la Constitución de la República Bolivariana de Venezuela (1999), como principal basamento legal del país, la cual será consultada para destacar los artículos que incidan en el presente estudio, tales como el artículo 102: "La educación es un derecho humano y un deber social fundamental, es democrática, gratuita y obligatoria..." (p.55).

Este artículo guarda relación con la presente investigación dado a que el mismo 
señala el derecho que tiene el niño a recibir educación en forma integral con la participación de la familia y la sociedad en un marco democrático y con el espíritu de solidaridad humana.

\section{MÉTODO}

El presente estudio se enmarcó dentro del tipo de investigación descriptivo, el cual según Hernández et al. (2006) señalan: "busca explicar las características más importantes de personas, grupos, comunidades o cualquier otro fenómeno que sea sometido a análisis, tomando la información directamente de la realidad en la que ocurre el hecho, fenómeno o situación" (p.26). Es por ello, que en esta oportunidad se analizaron la actitud hacia la física de los alumnos de 3er año del Ciclo Básico San Pablo estado Yaracuy; describiendo los detalles de la población estudiada.

Por otra parte, la investigación presentó un carácter no experimental, transeccional; al respecto Arias (2012) refiere que en los diseños no experimentales se observan fenómenos tal y como se dan en su contexto natural para después analizarlo, razón por la cual el autor antes citado refiere que en este diseño el investigador no asigna valores a las variables, observándose los hechos tal y como ocurren en la realidad.

En este mismo orden de ideas, Hernández, et al. (2006) refieren que un diseño transeccional o transversal recolecta datos en un solo momento, en un tiempo único, siendo su propósito describir variables y analizar su incidencia e interrelación en un momento determinado.

En el estudio se identificaron los sujetos, un conjunto limitado o un componente previamente seleccionado del universo, para efectos de este estudio la población se conformó por 128 estudiantes distribuidos en cuatro secciones (A, B, C y D) distribuidos en 76 hembras y 52 varones cursantes del 3er del Ciclo Básico San Pablo.
Por otra parte, la muestra es la selección de una parte de la población para la cual serán válidos los resultados del estudio. De acuerdo con Hurtado (2012) "es un sub-conjunto representativo de la población" (p.86). Para efectos del presente estudio la muestra estuvo representada por 08 estudiantes cursantes del 3er año sección "A" del Ciclo Básico San Pablo.

Para el proceso de recolección, se utilizaron tanto técnicas como instrumentos que permitieron registrar la información necesaria para dilucidar aspectos relevantes de la temática abordada, por ello la técnica utilizada en esta investigación fue la encuesta, que según Hurtado (2012) consiste en:

\section{Obtener información a través} de preguntas a otras personas. Se diferencia de la entrevista porque en la encuesta no se establece un diálogo con el entrevistado y el grado de interacción es menor. Los instrumentos propios de la técnica de encuesta son: el cuestionario, la escala, la prueba de conocimiento y los test. (p.449)

Es por ello, la encuesta permitió recopilar la información necesaria y así estudiar la situación problemática existente como es el caso de distinguir la actitud hacia la física de los alumnos de 3er año del Ciclo Básico San Pablo estado Yaracuy.

En este sentido, Hurtado (2012) define el cuestionario como “...un instrumento que agrupa una serie de preguntas relativas a un evento o temática particular, sobre el cual el investigador desea obtener información" (p. 449). Para efectos de este estudio, la recolección de datos se efectuará mediante un cuestionario tomado de Parra y Santos (2014) de un cuestionario que está dirigido a los estudiantes de tercer año del Ciclo Básico San Pablo del Municipio Arístides Bastidas estado Yaracuy.

En la investigación no se llevó a cabo el proceso de validación debido a que el instrumento fue tomado del trabajo realizado 
por Parra y Santos (2014) quienes llevaron a cabo este proceso antes de calcular su confiabilidad y ser aplicado a la muestra.

Posteriormente se efectuaron los análisis estadísticos para relacionar las variables. Es decir, se realizó un análisis de estadística descriptiva para cada una de sus variables y luego se describió la relación entre éstas. El tipo de análisis que se efectuó es descriptivo para las variables tomadas individualmente $\mathrm{y}$ se describieron los datos obtenidos en una distribución de frecuencia.

Aplicando estadística descriptiva con énfasis en una distribución y análisis porcentual de lo observado y obtenido en relación a la disposición al interés de la muestra hacia la realización del estudio planteada. Balestrini (2006) refiere que las técnicas de análisis de datos, se consideran "al culminar la fase de recolección de la información, los datos, han de ser sometidos a un proceso de elaboración técnica, que permite recontarlos y resumirlos, antes de introducir el análisis diferenciado a partir de procedimientos estadísticos..." (p. 169).

En este sentido, los datos obtenidos con la aplicación del instrumento, se analizaron y se tabularon para ser presentados en cuadros de frecuencias, porcentajes y gráficos de barras, pudiendo establecer conclusiones en base a las respuestas emitidas por los encuestados en cada uno de los ítems.

Finalmente, las distribuciones de frecuencias se completaron agregando las frecuencias relativas, que son los porcentajes de casos en cada categoría y las frecuencias acumuladas, que son las que se van acumulando en cada categoría, desde la más baja hasta la más alta, las cuales se presentaron en gráficas. Una vez que los datos fueron codificados $y$ transferidos a una matriz, se procedió al análisis e interpretación de los resultados, que permitió elaborar las conclusiones y recomendaciones de la presente investigación.

\section{RESULTADOS Y DISCUSIÓN}

Los resultados obtenidos a través de la aplicación del cuestionario a la muestra seleccionada perteneciente al 3er año de educación Media General del Ciclo Básico San Pablo del Municipio Arístides Bastidas, Estado Yaracuy, detectando las debilidades y las causas que originan el problema divisado en dicha institución, para de esta manera establecer el cuerpo de conclusiones producto del desarrollo de la fase diagnóstica.

En este sentido, Arias (2012) refiere que "el propósito del análisis es resumir las observaciones llevadas a cabo en forma tal que proporcionen respuestas a las interrogantes de investigación" (p.169); es así como los datos se analizaron e interpretaron por ítems, haciendo referencia a los porcentajes obtenidos, en cada categoría de respuestas, de acuerdo a lo establecido en el cuadro de las variables.

Para determinar los componentes que comprende la actitud se utilizó un instrumento tipo encuesta estilo Likert, de 12 ítems y cinco alternativas de respuesta Totalmente de Acuerdo, De Acuerdo, Indeciso, En Desacuerdo y Totalmente en Desacuerdo.

La actitud de los estudiantes del tercer año de Educación Media General del Ciclo Básico San Pablo, evidencian que la frecuencia porcentual más alta fue para la alternativa Totalmente De Acuerdo con un porcentaje de $38.54 \%$, seguida por la alternativa De acuerdo con un porcentaje de $32.29 \%$, luego se ubica la alternativa Indeciso con un $18.75 \%$, mientras que el resto se encuentra ubicado entre en desacuerdo con un porcentaje de $7.3 \%$ y Totalmente en desacuerdo con un $3.12 \%$, por lo se concluye que la actitud que los estudiantes poseen hacia la física tiene una tendencia favorable, sin embargo con unos índices que no superan el 50\% lo que conlleva a buscar que estos educandos se sientan más identificados con esta asignatura. 
Por otra parte, con relación a la actitud de los estudiantes del 3er año de Educación Media General hacia la física por ítems, se observa que la tendencia más elevada se evidencia en la alternativa Totalmente de acuerdo en los ítems cuatro (4), cinco (5), seis (6), siete (7), el ocho (8), nueve (9) y once (11), de la misma manera prevalece la alternativa De acuerdo en los ítem uno (1) y dos (2) indicando que ante las proposiciones los estudiantes mantuvieron una postura positiva por lo cual se considera la tendencia es favorable, el resto de los ítem están en las alternativas Indeciso, en desacuerdo $\mathrm{y}$ Totalmente en desacuerdo en menores porcentajes; por lo que se considera que la actitud es positiva.

Analizando los resultados obtenidos $\mathrm{y}$ tomando en cuenta los objetivos planteados en la presente investigación, se puede concluir que la actitud de los estudiantes del tercer año de Educación Media General del Ciclo Básico San Pablo del Municipio Arístides Bastidas del Estado Yaracuy, posee una tendencia favorable hacia la Física, observándose en los componentes de la actitud se tiene lo siguiente:

Para el componente cognitivo en sus indicadores convicción y conocimientos; se demostró una actitud favorable ubicada en las tendencias Totalmente de Acuerdo y De Acuerdo; sin embargo existe una parte de la población estudiantil en las que prevalece una actitud negativa o desfavorable.

En el componente afectivo, su indicador sentimientos; se prevalece la tendencia a una actitud favorable en un mayor porcentaje, mientras que un pequeño porcentaje se mantiene desmotivado y sin identificarse definitivamente con el aprendizaje de la física. Finalmente en el componente conductual e indicador conducta, los estudiantes muestran una tendencia favorable pero menos marcada que los anteriores componentes, por lo que se evidencia una considerable parte de la población con una actitud desfavorable en cuanto a su conducta hacia la física.

Los procedimientos en una investigación consisten, según Orozco, Labrador y Palencia (2002), en “...explicar lo que se va a realizar para lograr el objetivo de investigación, cómo se hará y con quién se efectuará" (p. 64), por lo cual para llevar a cabo la presente investigación se realizaron los siguientes pasos:

1) Elaboración de cuestionario para aplicarlo a los estudiantes.

2) Validación del instrumento por medio del juicio de los expertos.

3) Aplicación del instrumento a la muestra en estudio.

4) Selección del grupo piloto.

5) Cálculo de la confiabilidad.

6) Se analizaron y tabularon los resultados obtenidos a través de uso de tablas y gráficos, determinándose de esta manera los estadísticos de la investigación.

7) Se elaboraron las conclusiones $y$ recomendaciones.

Se realizó un análisis de estadística descriptiva para cada una de sus variables y luego se describió la relación entre éstas. El tipo de análisis que se efectuó es una estadística descriptiva para las variables tomadas individualmente y se describieron los datos obtenidos en una distribución de frecuencia.

A los datos recabados se les aplicó la estadística descriptiva con énfasis en una distribución y análisis porcentual de lo observado y obtenido en relación a la disposición al interés de la muestra hacia la realización del estudio planteada. Balestrini (2006) refiere que las técnicas de análisis de datos, se consideran "al culminar la fase de recolección de la información, los datos, han de ser sometidos a un proceso de elaboración técnica, que permite recontarlos y resumirlos, 
antes de introducir el análisis diferenciado a partir de procedimientos estadísticos..." (p. 169).

Finalmente, las distribuciones de frecuencias se completaron agregando las frecuencias relativas, que son los porcentajes de casos en cada categoría y las frecuencias acumuladas, que son las que se van acumulando en cada categoría, desde la más baja hasta la más alta, las cuales se presentaron en gráficas. Una vez que los datos fueron codificados $y$ transferidos a una matriz, se procedió al análisis e interpretación de los resultados, que permitió elaborar las conclusiones y recomendaciones de la presente investigación.

\section{CONCLUSIONES}

Concluyendo, a los docentes se les sugiere adoptar el uso de estrategias que favorezca la actitud de los estudiantes hacia la física, favoreciendo así la adquisición de conocimientos, dominio de contenidos teóricosprácticos; así como una mejor disposición hacia la asignatura. Además de convertirse en mediadores, facilitadores $\mathrm{y}$ orientadores dejando a un lado los modelos tradicionalista de ser solo transmisores de información; lo cual dificulta la comprensión y el entendimiento de la física.

También se resalta la importancia de utilizar métodos, recursos y materiales que propicien el amor hacia el aprendizaje de la física en los estudiantes. En este sentido, realizar clases participativas que le permita a los educandos ser partícipes de su proceso formativos, lo que conlleva a clases más dinámicas.

Los estudiantes deben enfatizar su interés en el rendimiento académico en la física y así mostrar mayor disposición a participar en las clases de física, lo que le permitirá aclarar dudas y obtener un aprendizaje significativo; por lo cual se sugiere a los educandos apoyarse en videos y materiales audiovisuales para lograr una mejor comprensión de los aspectos experimentales de la física.

\section{REFERENCIAS}

Álvarez, Cuéllar, López, Adrada, Anguiano, Bueno, Comas y Gómez. (2014). Las actitudes hacia la clase de física del estudiantado de secundaria. Chile: Universidad de Concepción

Arias, F. (2012). Guía para la elaboración de proyectos de investigación. (3ª edición). Caracas: Episteme

Balestrini, M. (2006). Cómo se elabora un proyecto de investigación. Caracas: $\mathrm{BL}$ Consultores Asociados

Bandura, A. (1963). Social learning theory. New Jersey: Prentice Hall

Bronfenbrenner, U. (1987). La ecología del desarrollo humano. Barcelona: Paidós

Castellano, M. (2013). Proyectos de Investigación: Una Metodología para el aprendizaje Significativo de la Física en Educación Media. Caracas: Universidad Central de Venezuela

Constitución de la República Bolivariana de Venezuela. (1999). Gaceta Oficial de la República de Venezuela. № 36.860. Diciembre 30, 1999

Fensham, P. (2004). Beyond Knowledge: Other Scientific Qualities as Outcomes for School Science Education. En R.M. Janiuk y E. Samonek-Miciuk (Ed.), Science and Technology Education for a Diverse World - dilemmas, needs and partnerships. International Organization for Science and Technology Education (IOSTE) XIth Symposium Proceedings (pp. 23-25). Lublin, Polland: Maria CurieSklodowska University Press

González, Morales y Pino. (2012). La educación científica como apoyo a la movilidad social: desafíos en torno al rol del profesor secundario en la implementación de la indagación científica como enfoque pedagógico. Estudios Pedagógicos XXXV (1), 63-78

Hernández, Fernández y Baptista. (2006). Metodología de la Investigación. México: Mc Graw Hill

Hurtado, J. (2012). Metodología de la Investigación Holística. Caracas: Fundación Sypal - Fundacite 
Martínez. (2004). Los Proyectos didácticos y la ciencia. UPEL-IMPM Núcleo Urachiche

Morales, Mazzitelli y Olivera. (2015). La enseñanza y el aprendizaje de la Física y de la Química en el nivel secundario desde la opinión de estudiantes. 1 Instituto de Investigaciones en Educación en las Ciencias Experimentales. Argentina

Nachtigall. (1985). Como resolver el dilema de la enseñanza de la Física. En UNESCO. Nuevas tendencias en la enseñanza de la física Vol. 4

Orozco, C., Labrador, M. y Palencia, A. (2002). Metodología. Manual teórico Práctico de Metodología para tesistas, asesores, tutores y jurados de trabajos de investigación y ascenso. Venezuela: Ofimax de Venezuela

Parra y Santos. (2014). Actitud de los estudiantes hacia el aprendizaje de la física en Tercer Año de Educación Media General. Caso: Unidad Educativa Casa Don Bosco Del Municipio Naguanagua. Año Escolar 2013-2014. Universidad de Carabobo, Venezuela

Solbes, Monserrat y Furió. (2007). El desinterés del alumnado hacia el aprendizaje de la ciencia: implicaciones en su enseñanza. España

Solé, H. (2002). Las ludotecas. Juguetes y sociedad. Barcelona: Paidós

Vázquez, A. y Manassero, M. (2007). La relevancia de la educación científica. Palma de Mallorca: Servei de Publicacions de la Universitat de les Illes Balears. Conselleria d 'Economia, Hisenda i Innovació

Vigotsky. (1981). Historia del desarrollo de las Funciones Psíquicas Superiores. Cuba: Científico Técnica 